\title{
POSTERIOR MAXILLARY SOCKET PRESERVATION USING COLLAGENATED CORTICO-CANCELLOUS XENOGRAFT
}

\author{
Nora El Ghazawy*; Iman M Helmy** and Maha Hakam**
}

\begin{abstract}
The current study was conducted to evaluate posterior maxillary socket preservation using Prehydrated Collagenated Cortico-Cancellous Equine Xenograft (Osteobiol@) compared to normal socket healing. 22 posterior maxillary extraction sites, indicated for socket preservation and implant placement were included in this study. They were randomly divided into two equal groups; 1-Study group: in which the extraction socket was filled with the bone graft. 2-Control group: in which the extraction socket was left to heal without bone grafting. All patients underwent preoperative evaluation, the target tooth was extracted atraumatically and the alveolar ridge was preserved using Osteobiol Putty bone graft material in the study group cases. All patients underwent follow up for 9 months. During this period they were regularly examined clinically to monitor healing and assess for any complications. Nine months after the first operative phase, bone core biopsy was taken from all cases of both groups, followed by implant placement. Histologic and histomorphometric evaluation of the retrieved biopsies was performed to assess for the amount, type, percentage of new bone formation as well as the osteoblastic/osteoclastic ratio. No major intraoperative or postoperative clinical complications were encountered in both groups. Histologic and histomorphometric examination of the bone biopsies revealed new bone formation in both groups. Morphometric analysis showed that the mean area fraction of the total bone matrix and the lamellar bone area fraction were significantly higher in the study group than the control group. The osteoblastic/osteoclastic ratio was significantly higher in the study group than in the control group. The used grafting material could be successfully utilized for socket preservation in posterior maxilla with minimally invasive procedures without the use of a barrier membrane or a mucoperiosteal flap.
\end{abstract}

\section{INTRODUCTION}

The rate and degree of the pneumatization process after tooth loss may be influenced by roots that protrude into the sinus cavity which have thin cortical bone lining. During extraction this thin bone may break and dislocate thus allowing the sinus to expand towards the empty socket. Also, greater expansion has been noticed after molar extraction in comparison to premolar extraction. The reason may be the large defect left in the alveolar bone after the molar extraction which requires a longer healing time thus allowing the sinus to pneumatize..$^{(1)}$

\footnotetext{
* Post Graduate Student, Oral and Maxillofacial Surgery Department, Faculty of Dentistry, Cairo University

** Professor, Oral Pathology Department, Faculty of Dentistry, Ain Shams University

*** Professor, Oral and Maxillofacial Surgery Department, Faculty of Dentistry, Cairo University
} 
In long time persisting edentulism in the molar zone it is rare to observe enough bone quantity between the maxillary sinus and the alveolar crest. ${ }^{(2)}$ In severe cases of edentulism, the oral cavity might be separated from the sinus just by a thin bone lamella. (3)

The rate of bone tissue damage may be accelerated by aggressive maneuvers during extractions. ${ }^{(4)}$ Whereas surgical trauma resulting from extraction induces microtrauma to surrounding bone, which fastens bone remodeling. ${ }^{(5)}$ Resorption also increases in case of a mucoperiosteal flap elevation. ${ }^{(6)}$ Resorption pattern in the maxilla progresses faster (because of the greater vascular supply) than those in the mandible.(7) Residual ridge resorption in maxilla is upwards and inwards, as a result after several years maxilla becomes progressively smaller. ${ }^{(8)}$.

Maxillary posterior alveolar ridge resorption is considered a dual process inside and outside of the sinus floor. This resorptive pattern is chronic, gradually progressive and cumulative. This ends by poor bone quantity and quality in this area, including reduced height and width.

Various types of bone grafting materials have been suggested for augmentation of dental sockets. Bone grafting material helps the barrier membrane in keeping space and providing biocompatible matrix for new bone formation. A systematic review was conducted on ridge preservation techniques. Different methods of ridge preservation were identified. The most common technique was placing a bone graft in the socket, covered by a membrane in addition to flap advancement to achieve partial or complete primary closure. ${ }^{(9)}$

Xenogenic biomaterials have long been used as bone substitutes, as its anorganic structure (Natural Hydroxyapatite- nHA) provides a natural architectural matrix as well as an excellent source of calcium. ${ }^{(10)}$ Moreover, sharing features with human bone, that is, a similar morphology and a potential of being resorbed is a main advantage of xenografts. Its resorption is slow, and it can be still present after 18 months in situ. ${ }^{(11-12)}$

Deproteinized bovine bone mineral (DBBM) is the most commonly used xenograft. ${ }^{(13)}$ It is one of the most well documented bone substitutes with osteoconductive properties and well incorporation in bone tissue as shown in experimental and clinical studies. ${ }^{(12,14)}$ Histological studies had demonstrated integration of titanium dental implants in areas previously treated with bovine bone..$^{(15,16)}$

The aim of this study is to evaluate posterior maxillary socket preservation using Collagenated Cortico-Cancellous Xenograft (Osteobiol®).

\section{PATIENTS AND METHODS}

The study involved 22 posterior maxillary extraction sites in adult patients of both sexes, indicated for socket preservation, prior to implant placement. Patients were selected from the outpatients' clinic of the Oral and Maxillofacial Surgery Department, Faculty of Dentistry, Cairo University and the outpatients' dental clinic of AlDemerdash hospital, Ain Shams University

Age of patients ranged from 20 to 46 years. The need for extraction was confirmed by clinical and radiographic examination (periapical and panoramic radiographs). All patients were informed orally and in writing, and gave their written consent before inclusion. The protocol was approved by the ethics committee of the faculty of Dentistry, Cairo University.

Selected extraction sites were randomly divided into two equal groups: Group A (Study group): Comprising 11 extraction sockets, filled with prehydrated collagenated cortico-cancellous equine bone mix (Osteobiol), as a socket grafting material. Group B (Control group): Comprising 11 extraction sockets, left to heal without socket grafting, to serve as a control. 
The operative procedures included two phases: Phase I: Included extraction of the target tooth and alveolar ridge preservation using Osteobiol Putty ${ }^{\circledR}$ bone graft material in group $A$, and no socket grafting in group B. Phase II: Was 9 months from phase I. Bone core biopsy was taken from both groups followed by implant placement. Both phases were performed under local anesthesia. Postextraction instructions were given to the patient both orally and in writing and postoperative medications were prescribed.

Sutures were removed after 14 days. Patients were clinically assessed at the following intervals: 48 hours, 1 week, 2 weeks, 1 month, 3 months, 6 months and 9 months. Patients were evaluated for soft tissue healing, wound dehiscence, bleeding, infection, sinusitis or any abnormality.

Core biopsy specimen was taken from the site of the implant (from the center of the grafted/non grafted site) using trephine bur ( $2 \mathrm{~mm}$ in diameter) guided by the surgical stent. The length of the core was corresponding to the length of the implant as planned from the measurements taken from the $\mathrm{CBCT}$ at 9 months.

Sequential osteotomes were used till reaching the appropriate drill size for proper implant placement. Submerged implant type was used. Finally, the flap was readapted and sutured. Postoperative instructions and medications were prescribed. Construction of the fixed prosthesis was started 4-6 months after implant insertion.

The specimens were then put in $10 \%$ buffered formalin then were decalcified in a mixture of $10 \%$ HCL and 10\% Formic acid for 24 hours. After decalcification, the specimens were embedded longitudinally into paraffin. Paraffin blocks were cut into $5 \mu \mathrm{m}$ thick sections. Then, the sections were stained with H\&E. Stained sections for both study and control groups were examined by the light microscope to assess: presence of new bone formation, the type of bone, whether lamellar or woven, presence of residual graft material, osteoblastic and osteoclastic activity and presence of chronic inflammatory cells.

Histomorphometric assessment was performed on the H\&E stained sections for the following parameters: Percentage of the total bone matrix area, percentage of the lamellar bone area, percentage of the woven bone area and osteoblastic/ osteoclastic ratio. These parameters were measured in the most representative 6 fields, captured at a constant magnification. The means were calculated. Comparisons between the study and the control groups were performed regarding the above mentioned parameters. As well as the percentage of the lamellar and the woven bone were compared in each group.

\section{RESULTS}

The early post operative follow up course in the study group went without any complications regarding swelling, bleeding, infection, soft tissue healing or pain. Except for case (no:2) who reported headache and pain at the surgery side for 7 days post operative. The same patient mentioned having attack of involuntary sneezing on day 6 , after which symptoms of OAC developed. So the suture removal for this patient was delayed for extra 1 week, after which the signs and symptoms of OAC disappeared. At 48 hours and 1 week follow up visits, the sockets were seen to be full of graft material in most of the cases, or slightly at a lesser level, with minimal tenderness. At 2 weeks follow up visit, patients presented with sockets closed and no complains. The rest of the follow up was uneventful, the clinical observation of the grafted sites at 9 months, showed no signs of infection, exudation or fistula formation were noted. In the control group, the follow up period was uneventful for all cases, with no intra or post operative complications.

Histological interpretation of the collected specimens in the study group revealed numerous thick spicules of mature trabecular bone showing characteristic features of the lamellar type were evident. Osteoblasts were seen riming the newly 
formed bone and delineating the marrow spaces. Large number of osteoblasts in variable stages of entrapments in the newly formed bone could be observed, reversal lines were highly seen revealing the rhythmic pattern of bone formation with high rate of bone remodeling (Fig 1). The medullary spaces showed well vascularized connective tissue, consisted of collagenous fibers which were haphazardly arranged in a background with newly formed blood vessels, indicating a still going healing process. No signs of inflammation, necrosis or foreign body reaction were observed.

Remaining minute fragments of the graft material were evident in 3 of the 11 cases. These particles appeared in complete continuity with new bone tissue that resulted from the remodeling. Many osteocytes trapped in their mineralized matrix were present in the mature bone in close proximity to the biomaterial surface (Fig 2a, b \&c).

Histological interpretation of the collected specimens in the control group revealed few thinner bone trabeculae and small amounts of trabecular bone with clearly seen lamellae surrounding the marrow spaces. Bone was mostly composed of interconnecting areas of woven bone. Identified by its unorganized fibrillar pattern showing haphazardly oriented collagen fibers interspersed in a background matrix rich in newly formed blood vessels and newly formed collagen fibrils (Fig 3a \&b).

Scattered osteocytes were irregularly arranged within the bony matrix. Osteoblastic riming was seen lining the osteoid layer at certain locations.
The osteoclastic activity with focal bone erosions were seen more frequently in the control group than in the study group. Marked inflammation was seen in one of the control group cases (Fig 4).

Histomorphometric measurments revealed that the mean area fraction of the total bone matrix was $46.57 \%( \pm 12.50)$ in the control group as compared to $72.67 \%( \pm 9.72)$ in the study group. Mann Whitney test revealed that the percentage of the total bone matrix is significantly higher in the study than the control group $(\mathrm{P}=0.002)$. In the control group the mean area fraction of lamellar bone was $8.64 \%$ $( \pm 8.28)$, while that of the woven bone was $38.11 \%$ $( \pm 15.21)$. In the study group, the mean area fraction of lamellar bone was $59.22 \%( \pm 26.45)$, while that of the woven bone was $2.37 \%( \pm 1.92)$. Mann Whitney test revealed that the lamellar bone area fraction was significantly higher in the study than in the control group with $(\mathrm{P}=0.001)$. Conversely, the woven bone area fraction was found to be significantly lower in the study group than in the control group with $(\mathrm{P}=$ 0.001) Table 1.

A comparison between the lamellar and woven bone in each group separately was done using Wilcoxon sign rank test. It revealed that, the mean area fraction of the lamellar bone was significantly higher than the woven bone in the study group with $(\mathrm{P}=0.008)$. Reversely, it was significantly lower in the control group with $(\mathrm{P}=0.011)$. The osteoblastic / osteoclastic ratio was significantly higher in the study group by a mean $4.19( \pm 2.0)$ than in the control group by a mean $0.44( \pm 0.16),(\mathrm{P}<0.001)$.

Table (1): Comparison of the histomorphometric data in the study versus the control group.

\begin{tabular}{|c|c|c|c|c|c|c|}
\hline \multirow{2}{*}{\multicolumn{2}{|c|}{ Parameter }} & \multicolumn{2}{|c|}{ Study } & \multicolumn{2}{|c|}{ Control } & \multirow{2}{*}{ P-value } \\
\hline & & Mean & SD & Mean & SD & \\
\hline Bone matrix area & $\%$ & 72.67 & 9.72 & 46.57 & 12.50 & $0.002 *$ \\
\hline Lamellar bone area & $\%$ & 59.22 & 26.45 & 8.64 & 8.28 & $<0.001^{*}$ \\
\hline Woven bone area & $\%$ & 2.36 & 1.92 & 38.11 & 15.21 & $<0.001 *$ \\
\hline \multicolumn{2}{|c|}{ Osteoblastic/ Osteoclastic ratio } & 4.19 & 2.0 & 0.44 & 0.16 & $<0.001 *$ \\
\hline
\end{tabular}




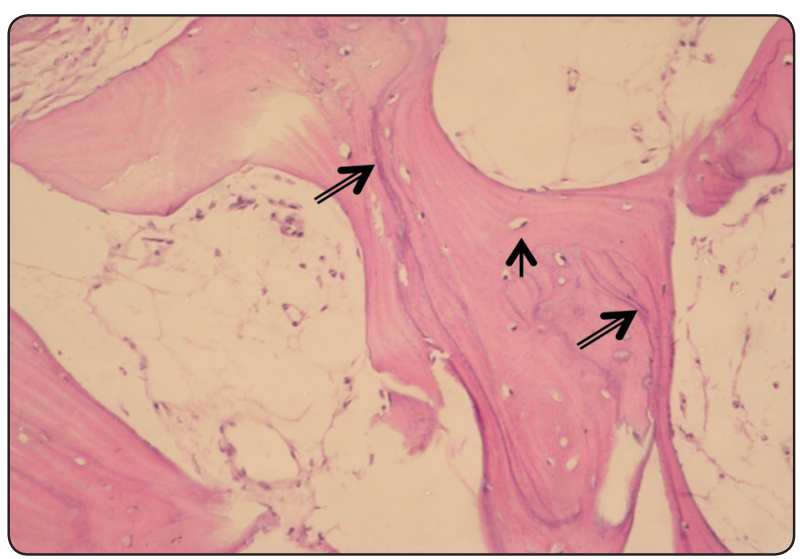

Fig(1): Photomicrograph of the study group showing thick trabeculae of lamellar bone, reversal lines ( $\Uparrow$ ) with osteocytes ( $\uparrow$ ) (H\&EX200).

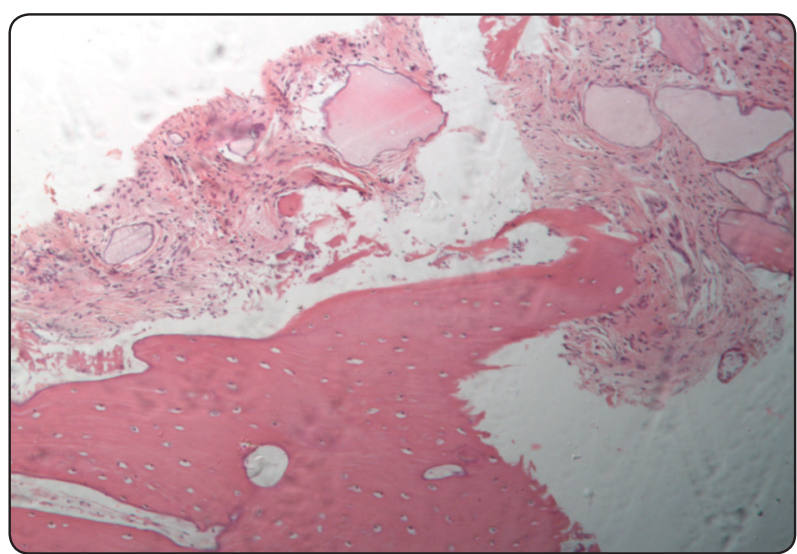

Fig(2b):The graft particles are surrounded by cellular connective tissue, showing osteoblast-like cells (H\& Ex200).

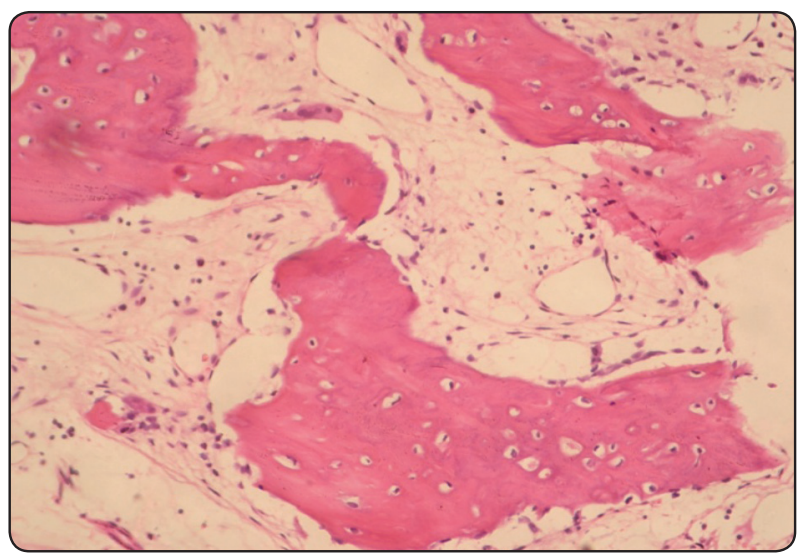

Fig (3a): Photomicrograph of the control group, showing thin trabeculae of woven bone (H\&EX200).

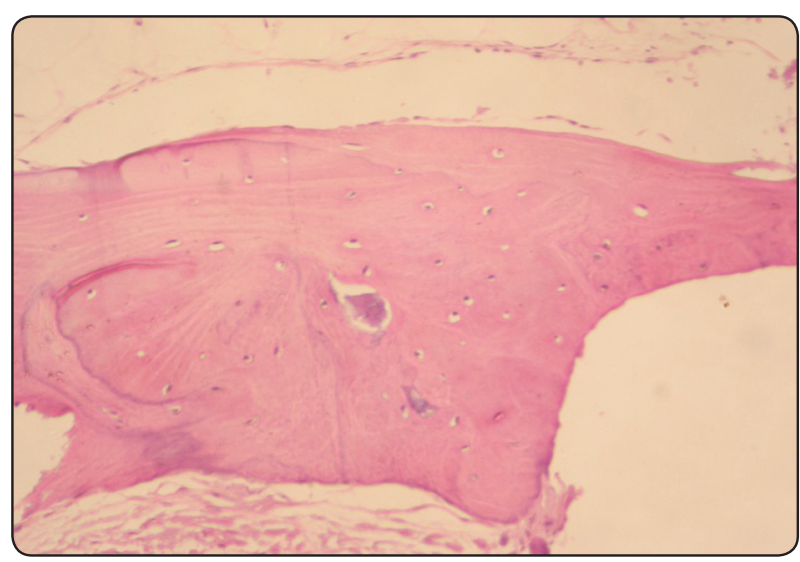

Fig(2a):Photomicrograph of the study group showing the residual bone graft particles embedded in a thick bone trabecula of newly remodeled lamellar type. Osteocytes and reversal lines are well demarcated (H\& Ex200).

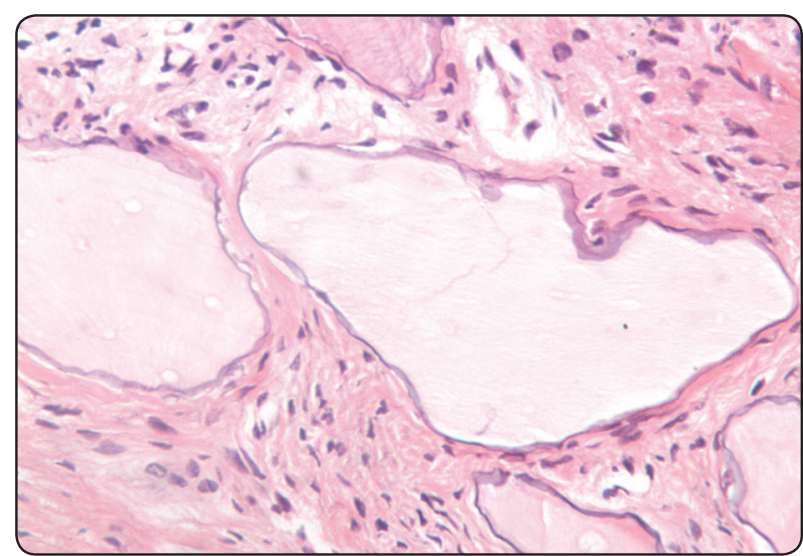

Fig(2c): The particles are presented with an amorphous structure, irregular outline and observed dark basophilic outline with a cement like appearance (H\&Ex400).

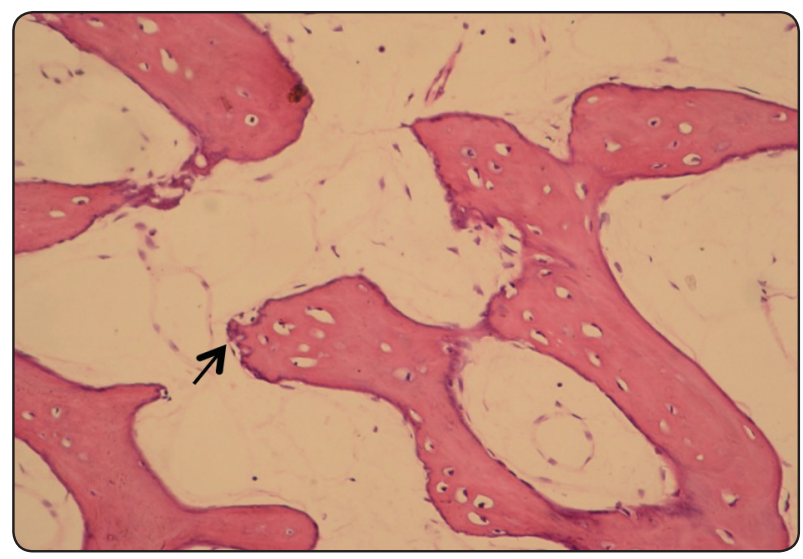

Fig. (3b): Photomicrograph of the control group, Multiple sites of erosion and osteoclasts $(\uparrow)$ were evident (H\&EX200). 


\section{DISCUSSION}

In the present study immediate posterior maxillary socket grafting was performed, in an attempt to preserve the horizontal and vertical dimensions of the alveolar ridge from the destructive sequalae of post- extraction resorption at different time intervals.

Patients were selected with the tooth to be extracted in close proximity to the maxillary sinus floor (the distance between the nearest point of the root and the sinus floor was $\leq 5 \mathrm{~mm}$. This critical primary insufficient bone volume was expected to significantly spotlight the effect of the preservation procedure versus the remodeling process that inevitably follows tooth extraction. Moreover, reducing the alveolar bone loss to acceptable levels by socket preservation, may omit the need for sinus lifting or at least allows the use of less invasive procedure (osteotome technique).

Intact socket walls (ie. 4-walls defect) was a main requirement in the included sockets, as the literature suggests that socket morphology has a critical impact on the outcomes of preservation efforts. ${ }^{(17)}$ During the clinical work, every effort was made to ensure atraumatic extraction, preserving both buccal and palatal plates and all the socket walls, as proposed by Iasella et al, 2003. ${ }^{(18)}$ The socket wall integrity was confirmed by direct probing during the surgical treatment.

Among different materials proposed for socket preservation, xenografts proved to be an interesting bone substitutes. Xenografts have similar chemical and structural composition of the autografts (mainly carbonated hydroxyapatite and type I collagen). Compared to the gold standard autogenous type, xenografts show morphological similarity (regarding bone surface, porosity and the potential of being resorbed), osteoconductivity and biocompatibility. Moreover, they shows superior advantages of being available in unlimited quantities with no donor site harmful effect. ${ }^{(12,14)}$ In the light of these values, xenograft was the grafting material chosen in the current work.
OsteoBiol ${ }^{\circledR}$ putty bone graft material in the current study exhibited no clinical adverse reactions and was shown to be well tolerated. This clinical finding was further confirmed by the histologic examination that proved no signs of inflammatory reaction around the graft particles. Same clinical and histologic results were obtained by many authors $^{(19,20)}$, who all confirmed the biocompatibility of OsteoBiol ${ }^{\circledR}$, being an antigen-free bone graft biomaterial.

While numerous socket preservation techniques are variable, the surgical technique used in this current study was standardized in order to decrease variability. Neither a flap nor a membrane was used to cover the post-extraction sockets in both groups (grafted and non grafted). Instead, it was left uncovered to heal spontaneously with secondary intention. The single interrupted suture done, was just to approximate the edges and reduce the amount of exposed material. Same surgical protocol was followed by many authors ${ }^{(21-23)}$ using an alloplastic materials for socket preservation of maxillary and/ or mandibular teeth. The selection of this (open socket approach) was in respect to different studies in the literature reporting that the use of flap and/ or membrane have no assured valuable importance.

Flapless, non-membrane technique was remarkably time saving, simple and comfortable to the patient. Postoperatively, most of the patients had uneventful healing showing no complications that are usually attributed to the surgical procedure complexity or prolonged surgery time. This goes in accordance with Camargo et al. ${ }^{(21)}$, who discouraged the use of regenerative procedures with flaps and membranes to allow simpler and less traumatic surgery.

Soft tissue coverage on top of the graft was observed to be completed 2 weeks postoperatively, which was comparable to the control group. Other studies that used the open socket approach ${ }^{(21-23)}$ had all reported the gradual lateral proliferation of the soft tissue to cover the defect in a period nearly the same of the current study. 
Different observations as the gradual soft tissue closure, the presence of the graft material in the surgical site until soft tissue complete closure and histologically proven well incorporated graft particles into the newly mineralized bone, implies that the graft shows primary stability that is sufficient to maintain it in place even if used in upper arch (anti-gravity) till epithalization occurs closing the exposed socket. Many factors may have a role in this primary stability, such as high particle density and the slow resorption rate of all xenografts. ${ }^{(24)}$ The presence of collagen may also enhance the handling properties and biomechanical characteristics of the material producing stable bone substitute.

Despite using this (open socket approach), the current study revealed new bone formation of acceptable quality and quantity after 9 months of healing as well as preserving socket width and height. Clinically the preserved ridge architecture appeared sufficient to achieve initial stability for subsequent implant placement. Same outcome was reported by Leventis et al. ${ }^{(23)}$ and Brkovic et

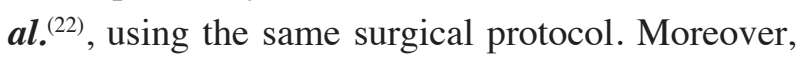
although reporting different histological appearance of bone samples in both membrane covered and non covered groups and the more apparent residual graft particles in the membrane group, yet Brkovic et al. ${ }^{(22)}$ reported similar histomorphometric results in both groups.

Not only routine histological evaluation was done, but also histomorphometrical analysis was performed. It worth mentioning that the use of the surgical stent for taking biopsies in this study, helped to take the bone biopsy from the center of the pre-existing sockets more accurately. So, that the specimens would not include original host bone.

The bone biopsies of the study group showed characters of newly formed bone, indicating a still going healing process. These findings are consistent with those of $\boldsymbol{A r t z i}$ et al. ${ }^{(24)}$, investigating xenograft preserved sockets after 9 months.
Histomorphometrical analysis of the current study demonstrated a significantly higher mean area fraction of the total bone in the study group, compared to the control group. These results are in accordance with Barone et $\boldsymbol{a l}^{(25)}$, who used the same graft material and examined their specimens at nearly the same timing postoperatively (7-9 months).

The distinct features of the woven bone as characterized by an unorganized morphology of collagen fibers, and the lamellar mature bone as presented in concentric/parallel layers containing collagen in organized parallel arrays, described by Artzi et al. ${ }^{(24)}$ could be observed in the present study. More importantly, the lamellar bone area fraction was significantly higher than the woven bone area fraction in the study group. This also agrees with Artzi et al $^{(24)}$ were they analyzed the amount of woven versus lamellar bone in sockets grafted with xenograft biomaterial, at 9 months postoperatively.

The significantly higher values of all measured parameters in the study cases compared to the controls, proved superior bone quality and quantity in the cases harvested the Osteobiol ${ }^{\circledR}$ Putty than the cases with natural healing. In addition, the significantly higher activity of osteoblasts shown in the study cases, demonstrated that active bone formation was still occurring 9 months following preservation and that the remodeling of immature woven bone into lamellar bone at the grafted sites was still ongoing. These results promoted the use of this xenograft material to fill the post extraction sites of posterior maxillary teeth, to avoid alveolar bone loss. Same conclusion was reported by Cardaropoli et al. ${ }^{(27)}$ and Nannmark and Azarmehr ${ }^{(28)}$, who all clearly demonstrated that the same collagenated cortico-cancellous bone mix (Osteobiol ${ }^{\circledR}$ Putty) induces bone formation.

Controversial results have been reported regarding the degree of resorption of the grafted material. In the present work, about two thirds of the cases showed resorption of the biomaterial at 
9 months postoperative, while only few residual particles were observed in the remaining cases. This is supported by the experimental study ${ }^{(29)}$ and the clinical work $^{(7)}$, using the OsteoBiol ${ }^{\circledR}$ graft biomaterial. These studies clearly demonstrated increased bone area with time in parallel with a resorption of this bone graft particles. As reported by Nannmark and Sennerby ${ }^{(29)}$, it is possible that this resorption is due to the presence of the collagen part in the material, inducing adhesion of osteoclasts to the surface of the material. Also, collagen has been shown to have a chemotactic and differentiation effect on mesenchymal stem cells.(30) Moreover, Arcuri et al. ${ }^{(31)}$, described the Osteobiol putty paste ( $80 \%$ granulated mix and $20 \%$ pure collagen) used in the current study to show complete resorption and substitution with trabecular bone tissue after only 3 months. Another recent experimental study ${ }^{(9)}$ evaluating the bone response to the OsteoBiol@ over a 4 months period, reported appreciable decrease in the total volume of the biomaterial demonstrating the bone grafts' partial and progressive resorption.

The bone biopsies of the study group showed characters of newly formed bone, indicating a still going healing process. These findings are consistent with those of Artzi et al. ${ }^{(24)}$, investigating xenograft preserved sockets after 9 months.

Histomorphometrical analysis of the current study demonstrated a significantly higher mean area fraction of the total bone in the study group, compared to the control group. These results are in accordance with Barone et al. $^{(25)}$, who used the same graft material and examined their specimens at nearly the same timing postoperatively (7-9 months).

The lamellar bone area fraction was significantly higher than the woven bone area fraction in the study group. This also agrees with Artzi et al. ${ }^{(24)}$ were they analyzed the amount of woven versus lamellar bone in sockets grafted with xenograft biomaterial, at 9 months postoperatively.
The significantly higher values of all measured parameters in the study cases compared to the controls, proved superior bone quality and quantity in the cases harvested the Osteobiol ${ }^{\circledR}$ Putty than the cases with natural healing. In addition, the significantly higher activity of osteoblasts shown in the study cases, demonstrated that active bone formation was still occurring 9 months following preservation and that the remodeling of immature woven bone into lamellar bone at the grafted sites was still ongoing. These results promoted the use of this xenograft material to fill the post extraction sites of posterior maxillary teeth, to avoid alveolar bone loss. Same conclusion was reported by Cardaropoli et al. ${ }^{(27)}$ and Nannmark and Azarmehr ${ }^{(28)}$, who all clearly demonstrated that the same collagenated cortico-cancellous bone mix (Osteobiol ${ }^{\circledR}$ Putty) induces bone formation.

Controversial results have been reported regarding the degree of resorption of the grafted material. In the present work, about two thirds of the cases showed resorption of the biomaterial at 9 months postoperative, while only few residual particles were observed in the other one third. This is supported by the experimental study ${ }^{(29)}$ and the clinical work $^{(32)}$, using the OsteoBiol ${ }^{\circledR}$ graft biomaterial. These studies clearly demonstrated increased bone area with time in parallel with a resorption of this bone graft particles. As reported by Nannmark and Sennerby ${ }^{(29)}$, it is possible that this resorption is due to the presence of the collagen part in the material, inducing adhesion of osteoclasts to the surface of the material.

According to Guirado et al..$^{(33)}$, this resorption have demonstrated the osteoconductive capacity of OsteoBiol ${ }^{\circledR}$, which acted as "scaffolding" for bone cells. In another study(24), osteoconductivity was determined via the promotion of osseous ingrowth and the close integration of the graft with the newly generated bone, which was further assuring the present observation. Using the Osteobiol ${ }^{\circledR}$ in his study, Barone et al. ${ }^{(34)}$ also supported the idea that it had excellent osteoconductive properties, and remodelling/resorption processes were taking place. 
The current study approved the spacemaintaining activity of the used graft material (heterologous cortico-cancelous bone mix 80\% and type I collagen 20\%). Osteobiol succeeded in better preservation of alveolar bone dimensions than the spontaneous normal socket healing, even in maxillary (antigravity) socket. The used grafting material could be successfully utilized for socket preservation in posterior maxilla with minimally invasive procedures without the use of a barrier membrane or a mucoperiosteal flap.

\section{REFERENCES}

1- Sharan A and Madjar D: Maxillary sinus pneumatization following extractions: A radiographic study. Int $\mathrm{J}$ Oral Maxillofac Impl 23: 48-56, 2008.

2- Watzek G: Endoosseous Implants: Scientific and Clinical Aspects. Chicago: Quintessence (publ Co), 1996.

3- Aghaloo T L and Moy P K: Which hard tissue augmentation techniques are the most successful in furnishing bony support for implant placement? Int J Oral Maxillofac Impl 22: 49-70, 2007.

4- Vanchit J, De Poi R and Blanchard S: Socket preservation as a precursor of future implant placement: Review of the literature and case reports. Compend Contin Educ Dent 28: 646-654, 2007.

5- Garetto L P, Chen J, Parr J A and Roberts W E: Remodeling dynamics of bone supporting rigidly fixed titanium implants: A histomorphometric comparison in four species including humans. Impl Dent 4: 235-243, 1995.

6- Fickl S, Zuhr O, Wachtel H, Bolz W and Huerzeler M: Tissue alterations after tooth extraction with and without surgical trauma: A volumetric study in the beagle dog. J Clin Periodontol 35: 356-363, 2008.

7- Soehren S E and Van Swol R L: The healing extraction site: A donor area for periodontal grafting material. J Periodontol 50: 128-133, 1979.

8- Manappallil J J: Complete Denture Prosthodontics. New Delhi, Arya (publ co), $1^{\text {sted, }} 2006$.

9- Darby I, Chen S T and Buser D: Ridge preservation techniques for implant therapy. Int J Oral Maxillofac Impl 24: 260-271, 2009.
10- Berglundh T and Lindhe J: Healing around implants placed in bone defects treated with Bio-Oss. An experimental study in the dog. Clin. Oral Impl Res 8: 117-124, 1997.

11- Froum S J, Wallace S S, Elian N, Cho S C and Tarnow D P: Comparison of mineralized cancellous bone allograft (Puros) and anorganic bovine bone matrix (Bio-Oss) for sinus augmentation: Histomorphometry at 26 to 32 weeks after grafting. Int J Periodontics Restorative Dent 26: 543 $551,2006$.

12- Klinge B, Alberius P, Isaksson S and Jonsson J: Osseous response to implanted natural bone mineral and synthetic hydroxyapatite ceramics in the repair of experimental skull bone defects. J Oral Maxillofac Surg 80: 241-249, 1992.

13- Artzi Z, Tal H and Dayan D: Porous bovine bone mineral in healing of human extraction sockets. Part 1: Histomorphometric evaluations at 9 months. J Periodontol 71: 1015-1023, 2000.

14- Fakuta K, Har-shai Y, Collares M V, Lichten J B and Jackson I T: Comparison of inorganic bovine mineral particles with porous hydroxyapatite granules and cranial bone dust in the reconstruction of full-thickness skull defects. J Craniofac Surg 3: 25-29, 1992.

15- Piattelli M, Favero GA, Scarano A, Orsini G and Piatelli A: Bone reactions to anorganic bovine bone (Bio-Oss) used in sinus augmentation procedures: A histologic long-term report of 20 cases in humans. Int J Oral Maxillofac Impl 14: 835-840, 1999.

16- Valentini P, Abensur D, Densari D, Graziani J N and Hämmerle C: Histological evaluation of Bio-Oss in a 2-stage sinus floor elevation and implantation procedure: A human case report. Clin Oral Impl Res 9: 59-64, 1998.

17- Horvath A, Mardas N, Mezzomo L A, Needleman I G and Donos N: Alveolar ridge preservation: A systemic review. Clin Oral Impl Investig 17: 341-363, 2013.

18- Iasella J M, Greenwell H, Miller R L, Hill M, Drisko C, Bohra A A and Scheetz J P: Ridge preservation using freeze-dried bone allograft and a collagen membrane compared to extraction alone for implant site development: A clinical and histologic study in humans. J Periodontol 74: 990-999, 2003.

19- Barone A, Aldini N N, Fini M, Giardino R, Guirado J L $\mathrm{C}$ and Covani $\mathrm{U}$ : Xenograft versus extraction alone for ridge preservation after tooth removal: A clinical and histomorphometric study. J Periodontol 79:1370-1377, 2008. 
20- Nannmark U and Azarmehr I: Short communication: Collagenated Cortico-Cancellous Porcine bone graft: A study in rabbit maxillary defects. Clin Impl Dent Relat Res 12: 161-163, 2010.

21- Camargo P M, Lekovic V, Weinlaender M, Klokkevold P R, Kenney E B, Dimitrijevic B, Nedic M, Jancovic S and Orsini M: Influence of bioactiveglass on changes in alveolar process dimensions after exodontia. Oral Surg Oral Med Oral Pathol Oral Radiol Endod 90: 581-586, 2000.

22- Brkovic B M B, Prasad H S, Rohrer M D, Konandreas G, Agrogiannis G, Antunovic D and Sándor G K B: Beta-tricalcium phosphate/type I collagen cones with or without a barrier membrane in human extraction socket healing: Clinical, histologic, histomorphometric and immunohistochemical evaluation. Clin Oral Invest 16: 581-590, 2012.

23- Leventis M D, Fairbairn P and Horowitz R A: Extraction site preservation using an in-situ hardening alloplastic bone graft substitute. Compend Contin Educ Dent 35: 1113,2014 .

24- Artzi Z, Tal H and Dayan D: Porous bovine bone mineral in healing of human extraction sockets: 2. Histochemical observations at 9 months. J Periodontol 72: 152-159, 2001.

25- Barone A, Aldini N N, Fini M, Giardino R, Guirado J L $\mathrm{C}$ and Covani $\mathrm{U}$ : Xenograft versus extraction alone for ridge preservation after tooth removal: A clinical and histomorphometric study. J Periodontol 79: 1370-1377, 2008 .

26- Brkovic B M B, Prasad H S, Konandreas G, Milan R, Antunovic D, Sándor G K B and Rohrer M D: Simple preservation of a maxillary extraction socket using betatricalcium phosphate with type I collagen: Preliminary clinical and histomorphometric observations. JCDA 74: 523-528, 2008
27- Cardaropoli D and Cardaropoli G: Preservation of the postextraction alveolar ridge: A clinical and histologic study. Int J Periodontics Restorative Dent 28: 469-477, 2008 .

28- Azarmehr I and Nannmark U: Short communication: Collagenated Cortico-Cancellous Porcine bone graft: A study in rabbit maxillary defects. Clin Impl Dent Relat Res 12: 161-163, 2010.

29- Nannmark U and Sennerby L: The bone tissue responses to prehydrated and collagenated cortico-cancellous porcine bone grafts: A study in rabbit maxillary defects. Clin Impl Dent Relat Res 10: 264-270, 2008.

30- Salasznyk R M, Williams W A, Boskey A, Batorsky A and Plopper G E: Adhesion to vitronectin and collagen I promote osteogenic differentiation of human mesenchymal stem cells. J Biomed Biotechnol 1: 24-34, 2004.

31- Clozza E, Biasotto M, Cavalli F, Moimas L and Di Lenarda R:Three-dimensional evaluation of bone changes following ridge preservation procedures.Int J Oral Maxillofac Impl 27: 770-775, 2012.

32- Barone A, Crespi R, Aldini N N, Fini M, Giardino R and Covani U: Maxillary sinus augmentation: Histologic and histomorphometric analysis. Int J Oral Maxillofac Impl 20: 519-525, 2005.

33- Guirado J L C, Fernandez M P R, Negri B, Ruiz R A D, Sanchez De-Val J E M and Moreno G G: Experimental Model of Bone Response to Collagenized Xenografts of Porcine Origin(OsteoBiol ${ }^{\circledR}$ mp3): A Radiological and Histomorphometric Study.Clin Impl Dent Relat Res 15: 143-151, 2013.

34- Barone A, Ricci M, Covani U, Nannmark U, Azarmehr I and Calvo-Guirado J L: Maxillary sinus augmentation using prehydrated corticocancellous porcine bone: Hystomorphometric evaluation after 6 months. Clin Impl Dent Relat Res 1-7, 2010. 